\title{
STUDIES OF HYPERFINE STRUCTURE OF LaI BY LASER SPECTROSCOPY ON ATOMIC BEAM
}

\author{
A. Kajoch, A. Krzykowski, D. Stefańska, B. Furmann and A. Jarosz \\ Institute of Physics, Poznań Technical University \\ Piotrowo 3, 60-965 Poznan, Poland
}

(Received May 23, 1995; revised version December 5, 1995)

\begin{abstract}
High-resolution Doppler-reduced laser spectroscopic investigations on an atomic beam of ${ }^{139} \mathrm{La}$ were carried out to study hyperfine structure of 8 odd levels. The magnetic-dipole coupling constants $A$ and the electric-quadrupole coupling constants $B$ were determined and compared with the data from literature, if a vailable.
\end{abstract}

PACS numbers: $35.10 . F k$

\section{Introduction}

The first works concerning the electronic (fine and hyperfine) structure of lanthanum atom were conducted already in the twenties and thirties of this century.

Meggers [1] in 1932 performed first measurements of a large number of spectral lines of lanthanum atom; on the basis of those measurements Russell and Meggers [2] carried out identification of many electronic levels, mostly belonging to the low-lying configurations; this identification has in most cases been confirmed in later works.

The first theoretical works, concerning the even configurations, were conducted by Stein [3]. The assignment of the theoretically determined electronic levels to the measured ones, for the even configurations, was performed by Wilson [4] and Ben Ahmed et al. [5, 6], who used the least squares method.

Anderson [7] was in 1934 the first to investigate the hyperfine structure of the isotope 139 of lanthanum atom, using a combination of a spectrograph and a Fabry-Perot interferometer. The continuation of those measurements were the works of Murakawa and Kamei [8], Lührs [9] and Fischer et al. [10], while Ben Ahmed [11] used Fourier spectroscopy. All of those investigations, performed by means of the classical optical spectroscopy, were of a rather limited accuracy.

The first precise measurements of the hyperfine structure of the levels belonging to the ground term were performed by Ting in 1957 [12] with the use of the atomic beam magnetic resonance (ABMR) method. The magnetic-dipole $(A)$, electric-quadrupole $(B)$ and magnetic-octupole $(C)$ hyperfine splitting constants were obtained. 
The precise measurements for the higher-lying metastable levels were carried out by Childs and Goodman in 1971 [13] — with the use of the ABMR method, and by Childs and Nielsen in 1988 [14] — with the use of the double optical-rf resonance on an atomic beam (ABMR-LIRF).

Investigations of the hyperfine structure of the electronic levels belonging to both even and odd configurations of lanthanum atom were also performed by various authors with the use of the following experimental methods:

- level-crossing spectroscopy (Hese [15]),

- laser-induced fluorescence on an atomic beam (Childs and Goodman $[16,17]$, Childs and Nielsen [14]),

- laser spectroscopy on an atomic beam with the detection via two-photon ionization (Shaw et al. [18]),

- laser optogalvanic spectroscopy (Behrens and Guthöhrlein [19], Govindarajan and Pramila [20], Pramila [21], Caiyan et al. [22] and Jia et al. [23]),

- laser Doppler-free two-photon spectroscopy in a hollow cathode (Jia et al. [24]).

Theoretical works concerning interpretation of the hyperfine structure of the even configurations were carried out, among the others, by: Childs and Goodman [13] - with the use of the Sandars and Beck effective operator theory [25], Childs and Nielsen [14] - with the use of the multiconfiguration theory of DiracFock, and Ben Ahmed et al. [5], who used the wave functions in intermediate coupling in order to calculate the angular part of the hyperfine interaction.

On having analyzed the papers concerning the hyperfine structure of the isotope ${ }^{139} \mathrm{La}$ which have been published so far, one can conclude that the existing interpretation of the hyperfine structure of the levels belonging to the even configurations, performed by Childs and Nielsen [14] for configurations $(5 d+6 s)^{3}$ only, is rather unsatisfactory. The possible reason is a strong perturbing influence of a high-lying $\left(\approx 40000 \mathrm{~cm}^{-1}\right)$ configuration $5 d 6 p^{2}$ on the $(5 d+6 s)^{3}$ system. Because of the position of the $5 d 6 p^{2}$ configuration in the energy scheme, its direct investigation would be connected with serious experimental problems. However, its interaction with the $(5 d+6 s)^{3}$ system can be simulated, if one can extract the information about the interaction of the $6 p$ electron with the nucleus from some other source. This would become possible if systematic precise measurements for the odd configuration $5 d^{2} 6 p$ were performed.

There are also many gaps in the existing experimental data for the levels belonging to the odd configurations $\left(5 d^{2} 6 p\right.$ and others), which has made so far performing the detailed analysis of the hyperfine structure of these levels impossible.

This situation induced us to carry out laser-spectroscopic investigations of the levels belonging to the odd configurations of lanthanum atom.

\section{Experimental}

The reported investigations of the hyperfine structure of lanthanum atom have been performed by the method of laser-induced fluorescence on an atomic beam. The schematic of the setup used is presented in Fig. 1 . 


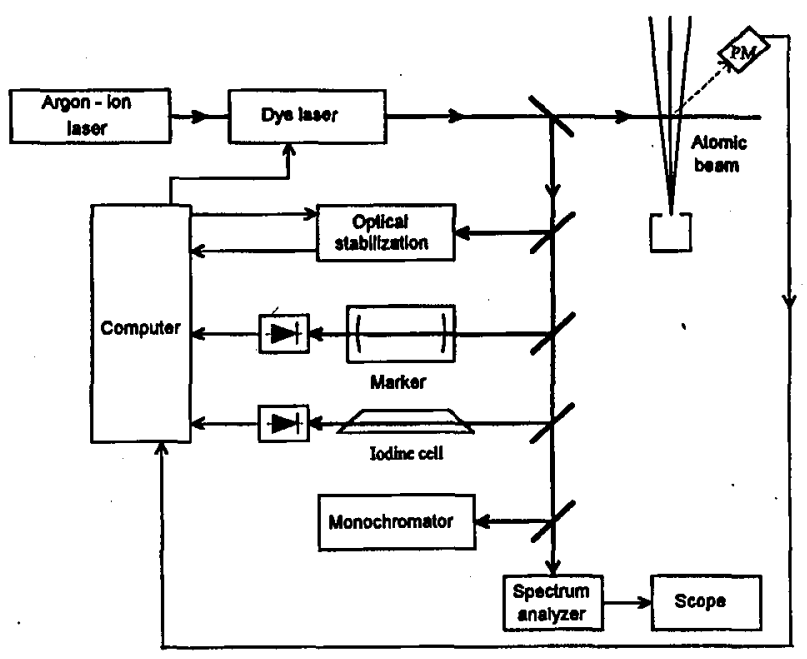

Fig. 1. Experimental setup used for investigations of the hyperfine structure with the method of laser induced fluorescence on an atomic beam.

All investigations have been carried out with the use of lanthanum metal samples of a natural composition $\left(99.911 \%{ }^{139} \mathrm{La}\right.$ and $\left.0.089 \%{ }^{138} \mathrm{La}\right)$.

The source of the radiation inducing optical transitions in lanthanum atoms was a cw tunable single-mode ring dye laser, constructed in the laboratory for laser spectroscopy of the Atomic Physics Division of the Institute of Physics, Poznan Technical University. The dye laser has been operated on Rhodamine 6G; it has been optically pumped by an argon ion laser (Spectra-Physics, model 2035 or Carl-Zeiss-Jena, model ILA 190) with the pump wavelength $514.5 \mathrm{~nm}$. The dye laser provides the output radiation within the spectral range of $570-620 \mathrm{~nm}$ with the maximum output power of about $700 \mathrm{~mW}$ (single-mode). The resonator geometrical configuration is based on Spectra-Physics, model 380D.

The external active laser frequency stabilization and tuning system, constructed in our laboratory [26], is based, like the original one of Spectra-Physics, on two tunable Fabry-Perot interferometers. The entire stabilization and tuning process is computer-controlled; simultaneously the experimental data are registered; this means that the computer control allows the direct synchronization of the laser frequency tuning and the measurement process. The laser frequency is changed stepwise with a stepwidth adjusted according to the requirements.

The coarse adjustment of the laser wavelength to the value corresponding to . the optical transition under study can be performed with the use of a monochromator (accuracy of $1 \mathrm{~cm}^{-1}$ ). The use of an absorption cell filled with molecular iodine allows the direct absolute wavelength control during the laser frequency scans (via comparison of the spectrum observed with the one from the tables [27]). The single-mode operation is controlled by a mode analyzer. The precise control of the laser frequency changes within the scan range is provided by a frequency marker (a temperature stabilized vacuum confocal Fabry-Perot interferometer with the $F S R=149.98(4) \mathrm{MHz})$. 
The laser beam, suitably formed, enters the vacuum chamber through a Brewster window and crosses the atomic beam at a right angle.

A cylindrical vacuum chamber is pumped out by a three-step oil diffusion pump. In the lower part of the chamber the oven for generation of the atomic beam, enclosed in a water jacket, is placed. The most essential part of the oven is a tantalum crucible containing a few grams of the element investigated. In the case of lanthanum (melting temperature $T_{\mathrm{m}}=920^{\circ} \mathrm{C}$ ) crucibles of $20 \mathrm{~mm}$ height and $10 \mathrm{~mm}$ diameter, closed with a tightly fitting cap provided with a hole of $0.8 \mathrm{~mm}$ diameter, were routinely used. Above the crucible a tungsten coil is placed, which is heated with a DC current up to about $2500^{\circ} \mathrm{C}$; a high voltage between the coil and the crucible causes the electrons emitted by the coil to flow towards the crucible - due to this electron bombardment the crucible is heated. Routinely the electron currents of $150-500 \mathrm{~mA}$ were obtained at the voltages of 1500-300 V, respectively. Some of the emitted electrons collide with the exiting crucible evaporated atoms of the element investigated. As a result higher-lying metastable levels are populated.

Above the water jacket a system of diaphragms for collimation of the atomic beam is placed. The diaphragms serve simultaneously to reduce the scattered light from the oven in the upper part of the vacuum chamber.

The atoms moving within the interaction zone of the atomic beam and the laser beam have a small velocity component which is perpendicular to the propagation direction of the atomic beam and parallel to the laser beam; this component causes Doppler broadening of the absorption lines observed in the excitation spectrum. For lanthanum atoms, under the conditions of generation of the atomic beam described above and within the spectral range in question, Doppler broadening amounts to $c a .5 \mathrm{MHz}$.

The fluorescence light, induced by the laser radiation, is collected by an optical detection system and, in the form of a slightly convergent beam, it is directed through a quartz window in the vacuum chamber onto the cathode of a photomultiplier (Hammamatsu, type R 375). Between the window in the vacuum chamber and the photomultiplier in some cases absorption or interference filters have been inserted, serving for selection of certain fluorescence lines and simultaneous suppression of the background radiation in other spectral ranges (scattered laser beam, light emitted by the hot oven).

The amplified signals from the photomultiplier, from the photodiode monitoring absorption in the iodine cell and from the frequency marker are simultaneously registered by the computer.

In the case of the most intense hyperfine components of the spectral lines the signal-to-noise ratio amounted to 14-140; for other hyperfine components the relation of signal and noise was less advantageous, in extreme cases the useful signal was comparable to the noise level - the hyperfine components, for which that was the case, were not taken into account in the final analysis of the results obtained.

The halfwidths of the single hyperfine components of the observed atomic spectral lines were typically $15-30 \mathrm{MHz}$. The total halfwidths consisted of the natural widths of the transitions, the residual Doppler broadening and the laser linewidth (of the order of 1-2 MHz). 


\section{Analysis of the experimental data}

The identification of the hyperfine components of the observed spectral lines has been performed via comparison of the registered experimental spectrum to the one simulated by a computer. The suitable choice of the parameters (hyperfine splitting constants $A$ and $B$ with given quantum numbers $I$ and $J$ for both the lower and the upper levels, halfwidths of the components) allows obtaining the agreement between the simulated and the experimental spectrum; thus a coarse estimation of the $A$ and $B$ constants is performed.

A precise calculation of the hyperfine splitting constants $A$ and $B$ for both the lower and the upper levels requires a precise determination of the intervals between the hyperfine components of a given spectral line. For this purpose a computer program fitting of each of the components with a suitable Voigt or Lorentz profile, using the least squares method, has been applied.

Once the identification of the hyperfine components of the spectral lines studied and the determination of the intervals between them have been performed, it was possible (using the so-called Casimir formula [28]) to construct a redundant set of linear equations with either four unknowns: $A_{1}, B_{1}, A_{\mathfrak{u}}$ and $B_{\mathrm{u}}$ for the lower and the upper levels, respectively, or with two unknowns only: $A_{\mathrm{u}}$ and $B_{\mathrm{u}}$ for the upper level (where $A_{1}$ and $B_{1}$ were taken from the literature). The number of equations is dependent on the number of the measured independent intervals between the hyperfine components of the spectral line under study. The redundant sets of equations have been solved numerically (with the use of the least squares method).

As each spectrum was registered several times (typically $3-7$ times), the first approach has been to obtain the $A$ and $B$ constants as the weighed means of the values calculated for individual scans. Experimental uncertainties, calculated as the errors of the weighed means, have been found to be generally too low, since discrepancies between the individual $A$ and $B$ values within a series typically exceeded the errors assigned to them in the fitting procedure. Thus the errors from the fitting procedure have been found inadequate and ignored further on. The final $A$ and $B$ values have been calculated as arithmetic means and the errors assigned to them are the standard deviations. The frequency calibration (resulting from the uncertainty of the FSR value of the marker) amounts to $0.03 \%$ and in most cases has been found smaller than the statistical errors considered. In a few cases when the calibration error has been larger than the standard deviation, the former has been taken as the final error value.

\section{Results of the measurements and discussion}

The registered hyperfine structure of the spectral lines $\lambda=574.440 \mathrm{~nm}$ and $\lambda=598.235 \mathrm{~nm}$ of ${ }^{139} \mathrm{La}$ are presented in Fig. 2 (the patterns belonging to the isotope ${ }^{138} \mathrm{La}$ were not observed because of its very low natural abundance).

The results obtained are listed in Table I. The first column contains the wavelengths of all the investigated optical transitions, which served to determine the hyperfine splitting constants of the electronic levels of lanthanum atom, belonging to the odd configurations (upper levels). Since not all of the transitions are mentioned in the tables of spectral lines of lanthanum atom [29,30], the appropriate 


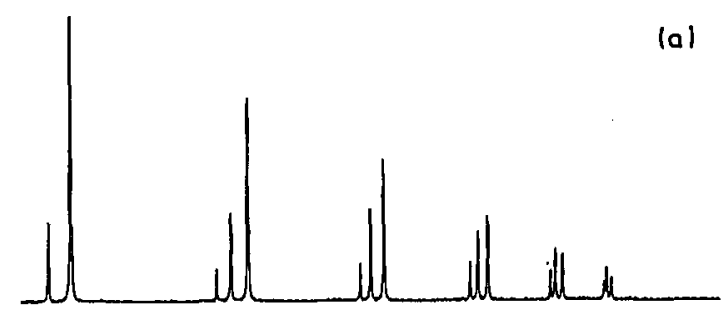

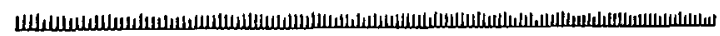

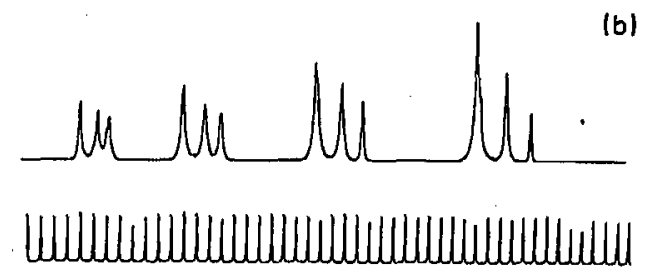

Fig. 2. The registered hyperfine structures of two spectral lines of ${ }^{139} \mathrm{La}$ : (a) $\lambda=$ $574.440 \mathrm{~nm}$, (b) $\lambda=598.235 \mathrm{~nm}$. Below the spectra the simultaneously recorded signals of the marker interferometer $(F S R=149.98(4) \mathrm{MHz})$ are presented.

wavelengths have been calculated as energy differences between the upper (investigated) and the lower levels. The designations and energy values of the upper and the lower levels are listed in the columns 2,3 and 4,5 , respectively.

The columns 6-9 contain the values $A_{\mathrm{l}}, B_{\mathrm{l}}, A_{\mathrm{u}}$, and $B_{\mathrm{u}}$, resulting from the four-parameter fit performed on the basis of our own results exclusively (fit 1 ), in the columns 10-13 the values $A_{\mathrm{u}}$ and $B_{\mathrm{u}}$ obtained in the two-parameter fit, where the literature values $A_{1}$ and $B_{1}$ from Refs. [12-14] have been taken as a constraint (fit 2), are listed. The comparison of the values $A_{1}$ and $B_{1}$ from fit 1 to the literature values, as well as the comparison of the values $A_{\mathbf{u}}$ and $B_{\mathbf{u}}$ from fit 1 and fit 2 to each other, show full agreement.

Table II contains a comparison of our results with the values obtained by other authors with the use of the following experimental techniques:

- a combination of a spectrograph and a Fabry-Perot interferometer [10],

- Fourier spectroscopy [11],

- laser optogalvanic spectroscopy (in the standard version and various modifications) [20-23].

Below the results of the comparison of the hyperfine splitting constants' values for 8 levels, obtained in this paper, to the results of other authors are presented in detail. In all cases the constants $B$ have been determined for the first time in the present paper, therefore only the constants $A$ can be compared to the literature data.

For the level $18172.35 \mathrm{~cm}^{-1}$ (No. 1) the value of the $A \cdot$ constant obtained in this paper agrees with the literature data $[10,11]$.

In the case of all the other levels the values of the constants $A$ disagree with 


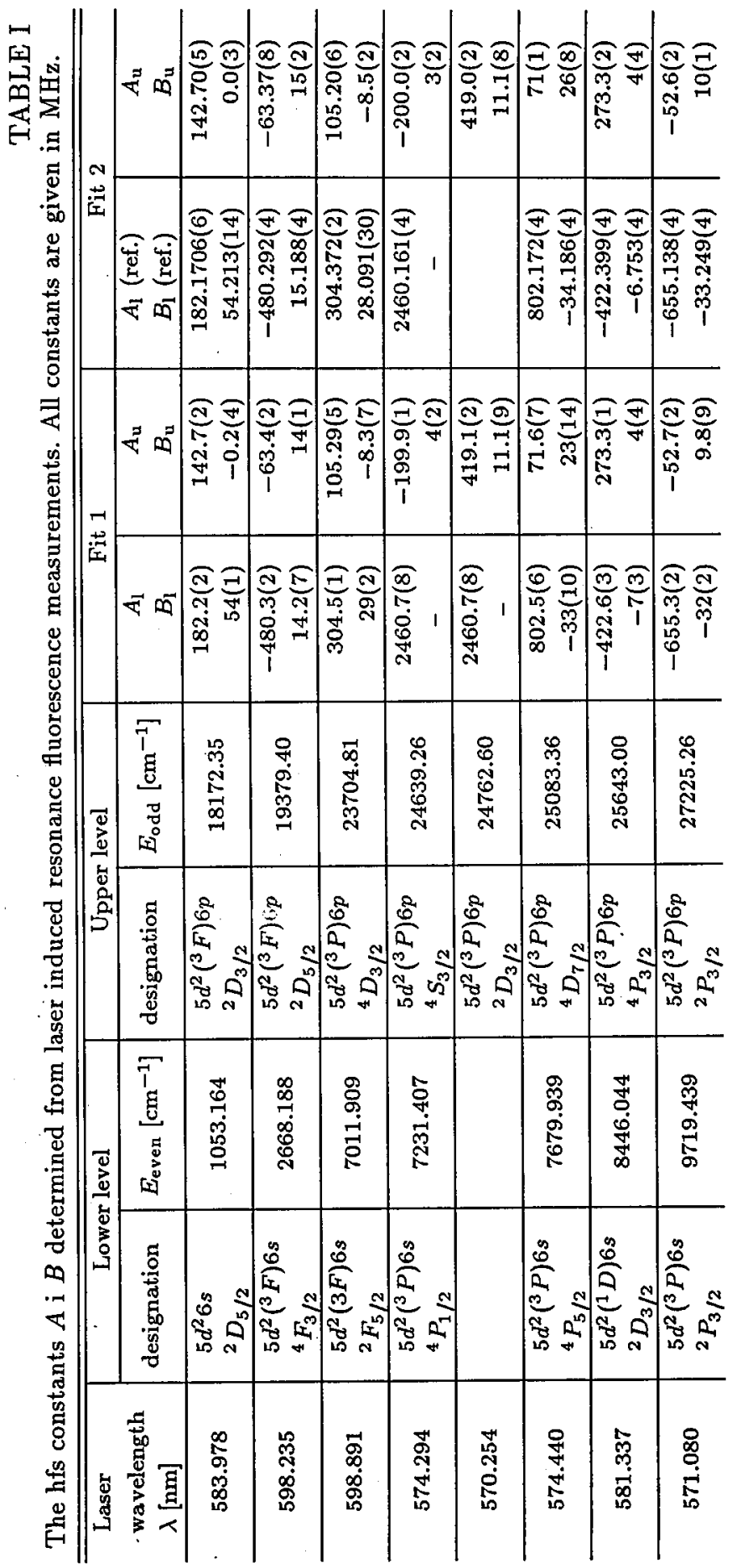


TABLE II

Comparison of the experimental hfs constants of the odd levels, obtained in this paper, with earlier results. The hfs constants are given in $\mathrm{MHz}$.

\begin{tabular}{l|r|r|r|r|r|l}
\hline \hline No. & $\begin{array}{c}\text { Level } \\
{\left[\mathrm{cm}^{-1}\right]}\end{array}$ & \multicolumn{2}{|c|}{$\begin{array}{c}\text { Experimental hfs } \\
\text { constants }\end{array}$} & \multicolumn{1}{|c|}{$A_{\text {ref }}$} & $B_{\text {ref }}$ & Ref. \\
\cline { 3 - 5 } & & \multicolumn{1}{|c|}{$A_{\mathrm{u}}$} & \multicolumn{1}{c|}{$B_{\mathrm{u}}$} & & & \\
\hline 1. & 18172.35 & $142.70(5)$ & $0.0(3)$ & $141(21)$ & - & {$[10]$} \\
2. & 19379.40 & $-63.37(8)$ & $15(2)$ & $-58.7(3)$ & - & {$[22]$} \\
3. & 23704.81 & $105.20(6)$ & $-8.5(2)$ & $117(9)$ & - & {$[11]$} \\
& & & & $156(6)$ & - & {$[20]$} \\
4. & 24639.26 & $-200.0(2)$ & $3(2)$ & $-232(6)$ & - & {$[20]$} \\
& & & & $-235(6)$ & - & {$[21]$} \\
5. & 24762.60 & $419.0(2)$ & $11.1(8)$ & $524(28)$ & - & {$[23]$} \\
6. & 25083.36 & $71(1)$ & $26(8)$ & $77(4)$ & - & {$[20]$} \\
& & & & $65(2)$ & - & {$[23]$} \\
7. & 25643.00 & $273.3(2)$ & $4(4)$ & $255(9)$ & - & {$[11]$} \\
& & & & $146(34)$ & - & {$[23]$} \\
8. & 27225.26 & $-52.6(2)$ & $10(1)$ & $-11(17)$ & - & {$[23]$}
\end{tabular}

the literature; however, there are two reasons for considering the values obtained in the present paper as reliable:

- the values of the constant $A$ have been calculated from the measurement of 5-12 independent intervals between the hyperfine components of the spectral lines registered several times,

- Table I contains a general verification of the results obtained in this paper, using as a basis very accurate literature data for the lower levels - this allows us to expect that the results are correct.

A detailed treatment for individual levels is presented below.

For the level $19379.40 \mathrm{~cm}^{-1}$ (No. 2) our value of the $A$ constant differs from the one given in [22] by less than $10 \%$; this difference exceeds, however, the error limits.

The value of the $A$ constant for the level $23704.81 \mathrm{~cm}^{-1}$ (No. 3) disagrees with the values given in [11,20]; in the case of the paper [20] the difference is essential $(\approx 50 \%)$. It should be stressed that the results of the papers [11] and [20] also do not show agreement between each other.

For the level $24639.26 \mathrm{~cm}^{-1}$ (No. 4) the value of the $A$ constant disagrees essentially $(15 \%)$ with the values given in $[20,21]$. Although the values from the mentioned papers agree with each other, the precision of the $A$ value in the present paper is two orders of magnitude better, which permits to believe that the reliability of the result obtained is higher. 
For the levels $24762.60 \mathrm{~cm}^{-1}$ (No. 5) and $27225.44 \mathrm{~cm}^{-1}$ (No. 8) the values of the $A$ constants disagree essentially (for the level No. $5: \approx 25 \%$, for the level No. $8: \approx 80 \%$ ) with respective values given in [23]; however, the precision of results obtained in the present paper is three orders of magnitude better than in [23].

The value of the $A$ constant for the level $25083.36 \mathrm{~cm}^{-1}$ (No. 6) obtained in this paper disagrees slightly (by less than $10 \%$ ) with both the values given in $[20,23]$. The constants in [20] and [23] also do not agree with each other. Our value lies between the two but the error limits do not overlap.

For the level $25643.00 \mathrm{~cm}^{-1}$ (No. 7) the value of the $A$ constant disagrees essentially with values given in $[11,23]$. Analogous to the case of level No. 6 the constant values given in $[11,23]$ disagree with each other.

\section{Conclusions}

To conclude the above discussion concerning hyperfine splittings constants - magnetic-dipole $A$ and electric-quadrupole $B$ for the various levels of the isotope ${ }^{139} \mathrm{La}$, let us note the following:

- for one level the value of the $A$ constant determined in this paper agrees with the value given before, for remaining 7 levels disagreements have been found, but the results obtained in this paper should be more reliable (because of better accuracy and a good reproducibility),

- for all the 8 levels the $B$ constants have been determined for the first time.

\section{Acknowledgments}

We wish to thank Prof. J. Dembczyński for inspiration to undertake these studies and many fruitful discussions and M.Sc. W. Kowalkiewicz for his expert technical assistance during the measurements.

This work was supported by the Project No. 2 P302 05204 (Committee for Scientific Research, Poland).

\section{References}

[1] W.J.F. Meggers, Res. Natl. Bur. Stand. (USA) 9, 239 (1932).

[2] H.N. Russell, W.J.F. Meggers, Res. Natl. Bur. Stand. (USA) 9, 625 (1932).

[3] J. Stein, J. Opt. Soc. Am. 57, 333 (1967).

[4]. M. Wilson, Phys. Rev. A 3, 45 (1971).

[5] Z. Ben Ahmed, C. Bauche-Arnoult, J.F. Wyart, Physica 77, 148 (1974).

[6] Z. Ben Ahmed, J. Verges, M. Wilson, A. Giacchetti, Physica C 84, 275 (1976).

[7] O.E. Anderson, Phys. Rev. 45, 685 (1934); ibid. 45, 473 (1934).

[8] K. Murakawa, T. Kamei, Phys. Rev. 92, 325 (1953).

[9] G. Lührs, Z. Phys. 141, 486 (1955).

[10] W. Fischer, H. Hühnermann, K. Mandrek, Z. Phys. 248, 53 (1971).

[11] Z. Ben Ahmed, Orsay, unpublished, 1976 .

[12] Y. Ting, Phys. Rev. 108, 295 (1957). 
[13] W.J. Childs, L.S. Goodman, Phys. Rev. A 3, 25 (1971).

[14] W.J. Childs, U. Nielsen, Phys. Rev. A 37, 6 (1988).

[15] A. Hese, Z. Phys. 236, 42 (1970).

[16] W.J. Childs, L.S. Goodman, J. Opt. Soc. Am. 67, 1230 (1977).

[17] W.J. Childs, L.S. Goodman, J. Opt. Soc. Am. 68, 1348 (1978).

[18] R.W. Shaw, J.P. Young, D.H. Smith, A.S. Bonanno, J.M. Dale, Phys. Rev. A 41, $2566(1990)$.

[19] H.O. Behrens, G.H. Guthöhrlein, J. Phys. C7 (France) 44, 149 (1983).

[20] J. Govindarajan, T. Pramila, J. Opt. Soc. Am. B 6, 1275 (1989).

[21] T. Pramila, Phys. Scr. 42, 556 (1990).

[22] L. Caiyan, Q. Jianan, Z. Lizhou, L. Fucheng, J. Phys. D 23, 1327 (1990).

[23] L.J. Jia, Ch. Jing, F. Lin, Opt. Commun. 94, 331 (1992).

[24] L. Jia, C. Jing, G. Guan, Z. Zhou, F. Lin, J. Opt. Soc. Am. B 10, 433 (1993).

[25] P.G.H. Sandars, J. Beck, Proc. R. Soc. 289, 97 (1965).

[26] A. Krzykowski, A. Kajoch, prepared for publication.

[27] S. Gerstenkorn, P. Luc, Atlas du spectre d'absorption de la molécule d'iode, Laboratoire Aime Cotton C.N.R.S. II, Orsay 1977.

[28] H. Kopfermann, Nuclear Moments, Academic Press, New York 1958.

[29] C.H. Corliss, W.R. Bozman, Experimental Transition Probabilities for Spectral Lines of Seventy Elements, Natl. Bur. Stand. (U.S.), Circ. 53, U.S. Government Printing Office, Washington D.C. 1962.

[30] A.N. Zajdel, W.K. Prokofiew, S.M. Rajskij, Tablicy spektralnykh linii, GITTL, Moskwa 1952.

[31] W.C. Martin, R. Zalubas, L. Hagen, Atomic Energy Levels - The Rare Earth Elements, Natl. Bur. Stand. (U.S.), Circ. 60, U.S. Government Printing Office, Washington D.C. 1978. 